\title{
Economic and genetic performance of various combinations of in vitro- produced embryo transfers and artificial insemination in a dairy herd
}

\author{
Karun Kaniyamattam, Jeremy Block, Peter J. Hansen, and Albert De Vries ${ }^{1}$ \\ Department of Animal Sciences, University of Florida, Gainesville 32611
}

\begin{abstract}
The objective of this study was to find the optimal proportions of pregnancies from an in vitro-produced embryo transfer (IVP-ET) system and artificial insemination (AI) so that profitability is maximized over a range of prices for embryos and surplus dairy heifer calves. An existing stochastic, dynamic dairy model with genetic merits of 12 traits was adapted for scenarios where 0 to $100 \%$ of the eligible females in the herd were impregnated, in increments of $10 \%$, using IVP-ET (ET0 to ET100, 11 scenarios). Oocytes were collected from the top donors selected for the trait lifetime net merit $(\mathrm{NM} \$)$ and fertilized with sexed semen to produce IVP embryos. Due to their greater conception rates, first ranked were eligible heifer recipients based on lowest number of unsuccessful inseminations or embryo transfers, and then on age. Next, eligible cow recipients were ranked based on the greatest average estimated breeding values (EBV) of the traits cow conception rate and daughter pregnancy rate. Animals that were not recipients of IVP embryos received conventional semen through AI, except that the top 50\% of heifers ranked for $\mathrm{EBV}$ of $\mathrm{NM} \$$ were inseminated with sexed semen for the first 2 AI. The economically optimal proportions of IVP-ET were determined using sensitivity analysis performed for 24 price sets involving 6 different selling prices of surplus dairy heifer calves at approximately $105 \mathrm{~d}$ of age and 4 different prices of IVP embryos. The model was run for $15 \mathrm{yr}$ after the start of the IVP-ET program for each scenario. The mean \pm standard error of true breeding values of $\mathrm{NM} \$$ of all cows in the herd in yr 15 was greater by $\$ 603$ \pm 2 per cow per year for ET100 when compared with ET0. The optimal proportion of IVP-ET ranged from ET100 (for surplus dairy heifer calves sold for $\geq \$ 300$ along with an additional premium based on their EBV of NM $\$$ and a $\leq \$ 100$ embryo price) to as low as ET0 (surplus dairy heifer calves sold at $\$ 300$ with a $\$ 200$
\end{abstract}

Received July 10, 2017.

Accepted September 24, 2017.

${ }^{1}$ Corresponding author: devries@ufl.edu embryo price). For the default assumptions, the profit/ cow in yr 15 was greater by $\$ 337, \$ 215, \$ 116$, and $\$ 69$ compared with ET0 when embryo prices were $\$ 50$, $\$ 100, \$ 150$, and $\$ 200$. The optimal use of IVP-ET was $100,100,62$, and $36 \%$ of all breedings for these embryo prices, respectively. At the input price of $\$ 165$ for an IVP embryo, the difference in the net present value of yr 15 profit between ET40 (optimal scenario) and ET0 was $\$ 33$ per cow. In conclusion, some use of IVP-ET was profitable for a wide range of IVP-ET prices and values of surplus dairy heifer calves.

Key words: in vitro production, embryo transfer, recipient, economics

\section{INTRODUCTION}

An in vitro-produced embryo transfer (IVP-ET) system can increase genetic progress by increasing the number of offspring produced from genetically elite females (Meuwissen, 1991; Kruip et al., 1994; Hansen, 2014). In this system, oocytes from genetically superior donors are harvested at a young age using ovum pickup procedures, fertilized in the laboratory, often with sexed semen, and cultured to produce genetically superior female embryos that are transferred to recipients. Major hurdles in the widespread commercial application of IVP-ET systems have been low laboratory efficiency (Chaubal et al., 2006; Block et al., 2010), high cost of producing transferable embryos (Hansen and Block, 2004; Wilson et al., 2006), and lower survivability of embryos as well as calves arising from the IVP-ET system (Bonilla et al., 2014).

Embryo transfer was found not to be profitable 30 yr ago (Ferris and Troyer, 1987). Ribeiro et al. (2012) found that the use of embryo transfer was only justifiable if the difference in fertility is large when compared with reproductive programs that exclusively used conventional semen. Heikkilä and Peippo (2012) found an optimal breeding mix that included conventional and sexed semen along with embryo donors with embryos sold to external farms for Finnish dairy herds. Beltrame et al. (2007) concluded that IVP of embryos using sexed semen reduced the cost per female pregnancy in dairy 
cattle. These 3 studies did not include the genetic gain from IVP-ET versus AI within the herd.

Ettema et al. (2011) and Heikkilä and Peippo (2012) advocated the need for using dynamic models that consider the genetic improvement over the course of several generations through selection in the herd to study more accurately the effect of various breeding types, including embryo transfer. Thomasen et al. (2016) illustrated the financial viability of using IVP-ET on the sire dam side of the Danish dairy population.

In a previous simulation study, we showed that a typical dairy herd using an exclusive IVP-ET system could sell up to $50 \%$ of its dairy heifer calves and still maintain an annual cow cull rate of approximately $33 \%$ (Kaniyamattam et al., 2017). Pryce et al. (2010) reported similar results. The breeding cost per cow per year was $\$ 405$ for an exclusive IVP-ET system after $15 \mathrm{yr}$ of implementation, compared with $\$ 46$ for an exclusive conventional AI system (Kaniyamattam et al., 2017). Even after accounting for the greater genetic value of the dairy heifer calves generated in the exclusive IVPET system and selling of surplus dairy heifer calves at a premium price that reflects their genetic merit, the exclusive IVP-ET system and the exclusive AI system were equally profitable in yr 15. Major financial losses per cow per year in the initial 3 yr accrued due to a delay in the return of investment in IVP embryos. A more optimal strategy could be to use IVP-ET to produce less than $100 \%$ of the pregnancies in the herd. Breeding with conventional or sexed semen would be used for animals that are not selected as embryo recipients.

Our hypothesis is that an optimal proportion of IVPET exists that includes a combination of IVP-ET and AI. The optimal proportion of IVP-ET likely depends on the prices of embryos and of surplus dairy heifer calves.

Our first objective was to describe the economic and genetic performance of dairy herds that use various proportions of pregnancies generated by IVP-ET and AI using a stochastic, dynamic dairy model with multitrait genetics. Our second objective was to perform a sensitivity analysis with 6 prices for surplus dairy heifer calves and 4 prices of embryos to estimate the economically optimal proportion of pregnancies that are achieved from IVP-ET.

\section{MATERIALS AND METHODS}

\section{Brief Description of the Model}

We used the original model of Kaniyamattam et al. (2016, 2017), with minor modifications made for this study. This is a daily dynamic stochastic model in which the genetic, technical, and financial performance of any animal in the herd is affected by 12 correlated genetic traits that are included in the 2014 net merit (NM\$) index (VanRaden and Cole, 2014). Genetic selection within the herd occurs by selling surplus dairy heifer calves not needed to replace culled cows, culling of low milk producing cows when heifers calve, and selective breeding with conventional or sexed semen or embryo transfer. Genetic trends for service sires are independent of the herd. The model simulates a herd of 1,000 milking cows with associated dry cows and young stock over time.

The original model included an exclusive IVP-ET system in which oocytes were collected from the top embryo donors ranked for $\mathrm{NM} \$$, and transferable embryos were transferred to selected recipients (Kaniyamattam et al., 2017). In the original model, all breedings were either exclusively with IVP embryos or with AI. For the current study, we modified the model to include changes in the use of donors and recipients so that a combination of both IVP-ET and AI could be simulated.

Donor Usage Pattern. We designed 11 scenarios with varying donor usage patterns that resulted in an increasing number of IVP embryos being produced (Table 1). The number of embryos produced in the herd in each 10-d interval was increased by 8 , so that the total of IVP-ET conceptions in the herd increased by approximately 10 percentage points, starting from a minimum of 0\% IVP-ET conceptions (ET0; all breedings with AI) to a maximum of $100 \%$ IVP-ET conceptions (ET100; all breedings with IVP-ET; Table 1). Donor selection occurred once every $10 \mathrm{~d}$ in all scenarios to have a constant supply of embryos over time. The initiation of the oocyte collection protocol occurred on different days within a 10-d window depending on the scenario (Table 1). For example, in the ET20 scenario, the oocyte collection procedure started for the first donor on $\mathrm{d} 1$, the second donor on $\mathrm{d} 4$, the third donor on d 7, and the fourth donor on d 9 .

Recipient Usage Pattern. The number of recipients was equal to the number of collections times 8 , starting from a low of 0 recipients for ET0 up to 160 for ET100 (Table 1). When the number of eligible recipients required was smaller than the total number of eligible animals available, we ranked the eligible recipients by expected probability of conception. Therefore, the eligible heifer recipients were first selected as recipients due to the greater conception rate for IVP embryos in heifers than in cows. Among the eligible heifer recipients, the ones with the lowest number of unsuccessful breedings and embryo transfers were ranked the highest. Second, the youngest of the eligible heifer recipients 
was first selected in case there was a tie for the number of unsuccessful breedings. Among the eligible cow recipients, the cows with the greatest average of EBV of daughter pregnancy rate (DPR) and cow conception rate (CCR) received embryos first. These animals were expected to have the highest conception rates (Kaniyamattam et al., 2017).

Recipients were selected on the same day the donors were selected. These possible recipients could be on any day of the estrous cycle and were observed for estrus daily with the probability of successfully detecting estrus being 50\% (Kaniyamattam et al., 2016). If estrus was observed in a selected recipient, the animal was scheduled to receive an IVP embryo on $\mathrm{d} 6,7$, or 8 after estrus, depending upon if a fresh embryo was first available. Frozen embryos were transferred in the event that fresh embryos were not available, similar to the procedure described in Kaniyamattam et al. (2017). All eligible animals, which were not selected as recipients, received AI as described in Kaniyamattam et al. (2016).

\section{Experimental Design}

Simulations. The simulations spanned 30 yr divided into 3 stages of $10 \mathrm{yr}(\mathrm{d}-5,475$ to $-1,875), 5 \mathrm{yr}$ (d $-1,874$ to 0 ), and 15 yr (d 1 to 5,475 ) as described in Kaniyamattam et al. (2017). Motivation for these periods was as follows. Initially, steady-state results

Table 1. Donor and recipient usage in 11 in vitro-produced embryo transfer (IVP-ET) scenarios simulated with the stochastic multi-trait simulation model

\begin{tabular}{llc}
\hline Name $^{1}$ & Donor usage pattern & No. of recipients $^{3}$ \\
\hline ET0 & $0-0-0-0-0-0-0-0-0-0$ & 0 \\
ET10 & $1-0-0-0-0-1-0-0-0-0$ & 16 \\
ET20 & $1-0-0-1-0-0-1-0-1-0$ & 32 \\
ET30 & $1-0-1-0-1-0-1-0-1-1$ & 48 \\
ET40 & $1-1-1-0-1-1-1-0-1-1$ & 64 \\
ET50 & $1-1-1-1-1-1-1-1-1-1$ & 80 \\
ET60 & $2-1-1-1-1-2-1-1-1-1$ & 96 \\
ET70 & $2-1-1-2-1-1-2-1-2-1$ & 112 \\
ET80 & $2-1-2-1-2-1-2-1-2-2$ & 128 \\
ET90 & $2-2-2-1-2-2-2-1-2-2$ & 144 \\
ET100 & $2-2-2-2-2-2-2-2-2-2$ & 160 \\
\hline
\end{tabular}

${ }^{1}$ Name of the scenario: $\mathrm{ET}=$ use of in vitro-produced embryos to obtain varying proportions of conceptions from IVP-ET, ranging from no use (ET0) to exclusive use (ET100) of IVP-ET, in increments of 10 percentage points. Animals that were not selected as embryo recipients were artificially inseminated with conventional or sexed semen.

${ }^{2}$ Number of donors required per day in a 10-d interval for a 1,000-milking cow herd with additional young stock to replace culled cows. Donors were selected once every $10 \mathrm{~d}$ based on the highest EBV of lifetime net merit.

${ }^{3}$ The number of recipients selected in a $10-\mathrm{d}$ interval for varying proportions of IVP-ET. were obtained after running the model for $15 \mathrm{yr}$ with conventional semen only and random culling of surplus dairy heifer calves every month. Results from the last 5 of these $15 \mathrm{yr}$ were retained. The 11 scenarios with various proportions of IVP-ET breedings were simulated in the final $15 \mathrm{yr}$, based on the same default steady state herd present on d 0 of the simulation. By yr 15 these 11 scenarios had reached the approximate steady state again such that annual changes were near constant.

The average true breeding value (TBV) of all traits of cows in the herd was approximately 0 on $\mathrm{d} 0$ following initial TBV values set on $\mathrm{d}-5,475$. Herd size was kept at 1,000 milking cows as in Kaniyamattam et al. (2017). Each scenario was replicated 100 times to generate standard errors as small as feasible given the speed of the Java code of this model and available hardware. Many output statistics were collected, including genetic, technical, and economic statistics. Twenty-one day pregnancy rates were calculated with a voluntary waiting period of $50 \mathrm{~d}$ for cows. The GLM procedure in SAS version 9.4 (SAS Institute Inc., Cary, $\mathrm{NC}$ ) was used for one-way ANOVA of output statistics among the scenarios.

Sensitivity Analysis. Sensitivity analysis was performed with 24 price sets to determine the optimal proportions of IVP-ET that maximized profit per cow per year in each set. Price sets were a combination of 3 base prices for sold surplus dairy heifer calves $(\$ 300, \$ 500$, and $\$ 700)$, with or without a premium price based on the sold dairy heifer calves' EBV of $\mathrm{NM} \$$, and 4 embryo prices $(\$ 50, \$ 100, \$ 150$, and $\$ 200)$. Surplus dairy heifer calves were sold at approximately $105 \mathrm{~d}$ of age, at which time rearing costs incurred since birth were $\$ 375$ (Kaniyamattam et al., 2017). The premium price was calculated as the product of a net present value (NPV) adjuster and the difference between $\mathrm{EBV}$ of $\mathrm{NM} \$$ of the average sold dairy heifer calf in the specific IVP-ET scenario and the ET0 scenario. We assumed that $85 \%$ of the dairy heifer calves born and kept became calving heifers and the expression of $\mathrm{NM} \$$ occurred an average $3.5 \mathrm{yr}$ after first calving. Assuming a 5\% annual interest rate, the NPV adjuster was $0.85 \times(1 / 1.05)^{3.5}=0.72$.

To find the optimal proportions of IVP-ET in each of the 24 price sets, a quadratic equation was fitted on the 3 consecutive scenarios with the highest profit per cow per year per price set. The highest point on the curve determined the optimal proportion of IVP-ET conceptions for each of these 24 scenarios. The maximum additional profit per cow per year from use of the optimal IVP-ET system was calculated as the difference between the profit per cow per year from the ET0 scenario (AI only) and that from the optimal IVP-ET system. 


\section{RESULTS}

\section{Default Starting Herd}

The average TBV of the 13 traits for cows in the herd for yr 0 are shown in Table 2. The aim was to obtain TBV close to 0 for all traits. Thus, the 11 IVP-ET scenarios were simulated in a herd starting with a $-\$ 4$ TBV of NM \$, 21\% pregnancy rate, $145 \mathrm{~d}$ open, and milk yield of $10,679 \mathrm{~kg} / \mathrm{yr}$. The annual cull rate was $31 \%$, and $8 \%$ of the dairy heifer calves born alive were culled as surplus calves.

\section{Genetic Herd Performance}

Table 2 also shows genetic performances in yr 15 after use of the 11 IVP-ET scenarios. Average TBV in yr 15 were different $(P<0.05)$ for all traits in almost all scenarios. The scenario ET100 resulted in the greatest average TBV in the direction of the desired change for all 13 traits. The TBV of milk in scenario ET100 increased by $2,477 \mathrm{~kg} / 305 \mathrm{~d}$ in $15 \mathrm{yr}$. The difference in average TBV of milk between ET100 and ET0 was 496 $\mathrm{kg} / 305 \mathrm{~d}$. Of this difference between 0 and 100\% IVPET, $73 \%(362 \mathrm{~kg})$ was obtained by generating $40 \%$ of pregnancies by IVP-ET (ET40). The rate of increase in TBV for all traits decreased as the proportion of IVPET increased. The greatest increase was obtained from ET0 to ET10 (142 kg milk in $305 \mathrm{~d}$, or $29 \%$ of $496 \mathrm{~kg}$ ).

The TBV of NM $\$$ increased by $\$ 2,984$ from yr 0 to 15 in the ET100 scenario. In yr 15, the TBV of NM\$ increased by $\$ 444$ (74\% of $\$ 603)$ when the proportion of pregnancies from IVP-ET was increased from 0 to $50 \%$. Therefore, the increase was only $\$ 169(26 \%)$ when the proportion of pregnancies from IVP-ET increased from 50 to $100 \%$.

The change in the TBV of $\mathrm{NM} \$$ over time is shown in Figure 1 for 6 scenarios. It is evident that the exclusive use of IVP-ET (ET100) resulted in the greatest TBV for $\mathrm{NM} \$$ from yr 3 onward, when the herd started to consist of some cows born from IVP-ET. Figure 1 also shows that the rate of increase in TBV of $\mathrm{NM} \$$ was the greatest between ET0 and ET20 and was reduced with higher proportions of IVP-ET. By yr 15, the genetic lag between the cows in the ET100 scenarios and the service sire was $\$ 373$ of TBV of $\mathrm{NM} \$$, a reduction of $66 \%(\$ 1085-\$ 373) / \$ 1085$ compared with the lag of $\$ 1,085$ in yr 0 .

Dairy heifer calves born in the ET100 scenario had the highest average EBV of $\mathrm{NM} \$$ (Figure 2) because all calves sold were generated from the top $2 \%$ of animals for EBV of NM\$ after yr 2. Genomic testing first started on the first day of yr 1 and the dairy heifer calves with the lowest EBV were identified with greater reliability. Therefore, the average EBV of $\mathrm{NM} \$$ of sold dairy heifer calves in yr 1 was lower than in yr 0 (Figure 2 ). The average EBV of surplus dairy heifer calves was $\$ 564$ higher in ET100 than in ET0 in yr 1 to 15 . The differences in the EBV of $\mathrm{NM} \$$ over time were used in the premium price calculation of sold surplus dairy heifer calves.

\section{Technical Herd Performance}

The actual proportion of pregnancies obtained from IVP-ET varied slightly from the intended proportion for all scenarios other than ET0 and ET100 (Table 2) because of the various donor usage patterns (Table 1 ). Cow 21-d pregnancy rates increased by at least 14 percentage points from yr 0 to 15 . In yr 15 , the 21 -d pregnancy rate increased by only 1 percentage point when the proportion of IVP-ET pregnancies was increased from 0 to $100 \%$, even though the combined change in TBV of DPR and TBV of CCR was 4.23 percentage points. An increase of 11 percentage points in abortions in cows and 8 percentage points in heifers was observed between the ET0 and ET100 scenarios because of the greater probability of abortion of IVP embryos. The increase in risk of abortion increased days open by 15 d (Table 2).

The average age of the dams of the cows in the herd calculated when they were conceived as embryos decreased by 597 d between ET0 (952 d) and ET100 (355 d). This reduction was due to the use of mainly heifers to produce transferable embryos. In yr 15 , the fraction of surplus dairy heifer calves sold was at least $30 \%$ (ET0) and increased to 54\% (ET100).

The annual cow cull rate did not change much between scenarios in yr 15 . The involuntary cull rate decreased from $16.0 \%$ in ET0 to $13.8 \%$ in ET100. The increase in the TBV of productive life by 5 mo between ET100 and ET0 contributed to the decrease in involuntary cull rate. This decrease in involuntary cull rate was nullified by the increase in rebalance culling whereby the cows producing the least milk were culled to make room for calving heifers to maintain 1,000 milking cows in the herd. Milk yield in $305 \mathrm{~d}$ increased by $490 \mathrm{~kg}$ from the ET0 and ET100 scenarios in yr 15, which is similar to the $496 \mathrm{~kg}$ per $305 \mathrm{~d}$ increase in the TBV of milk yield for these scenarios.

\section{Financial Herd Performance}

Revenues. In yr 15, the ET100 scenario had the highest milk sales $(\$ 5,768)$, dairy heifer and bull calf sales at the base price $(\$ 260)$, profit deviation $(\$ 83)$, and hence the greatest revenues $(\$ 6,427)$ per cow per year (Table 3). The highest revenues in ET100 cor- 


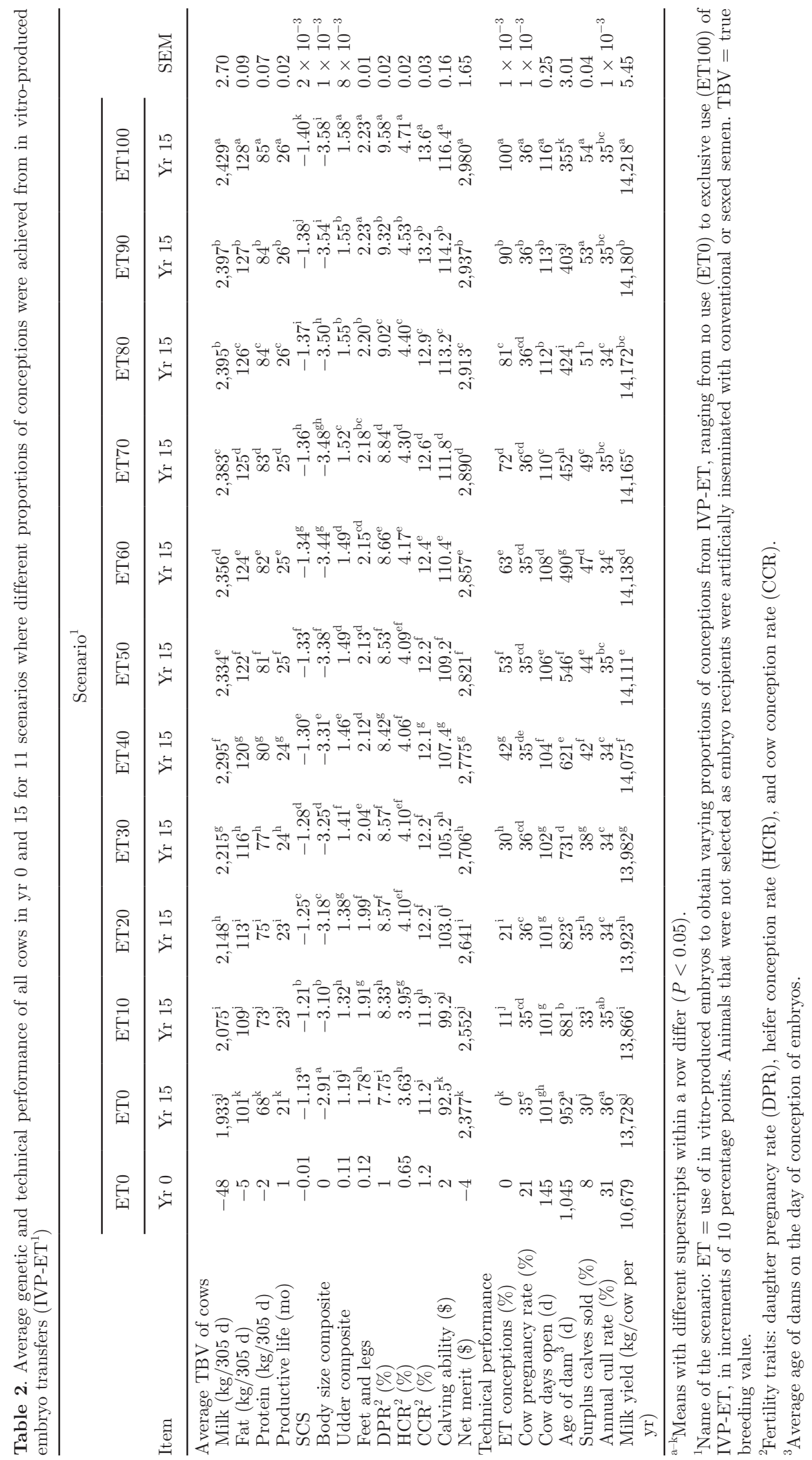




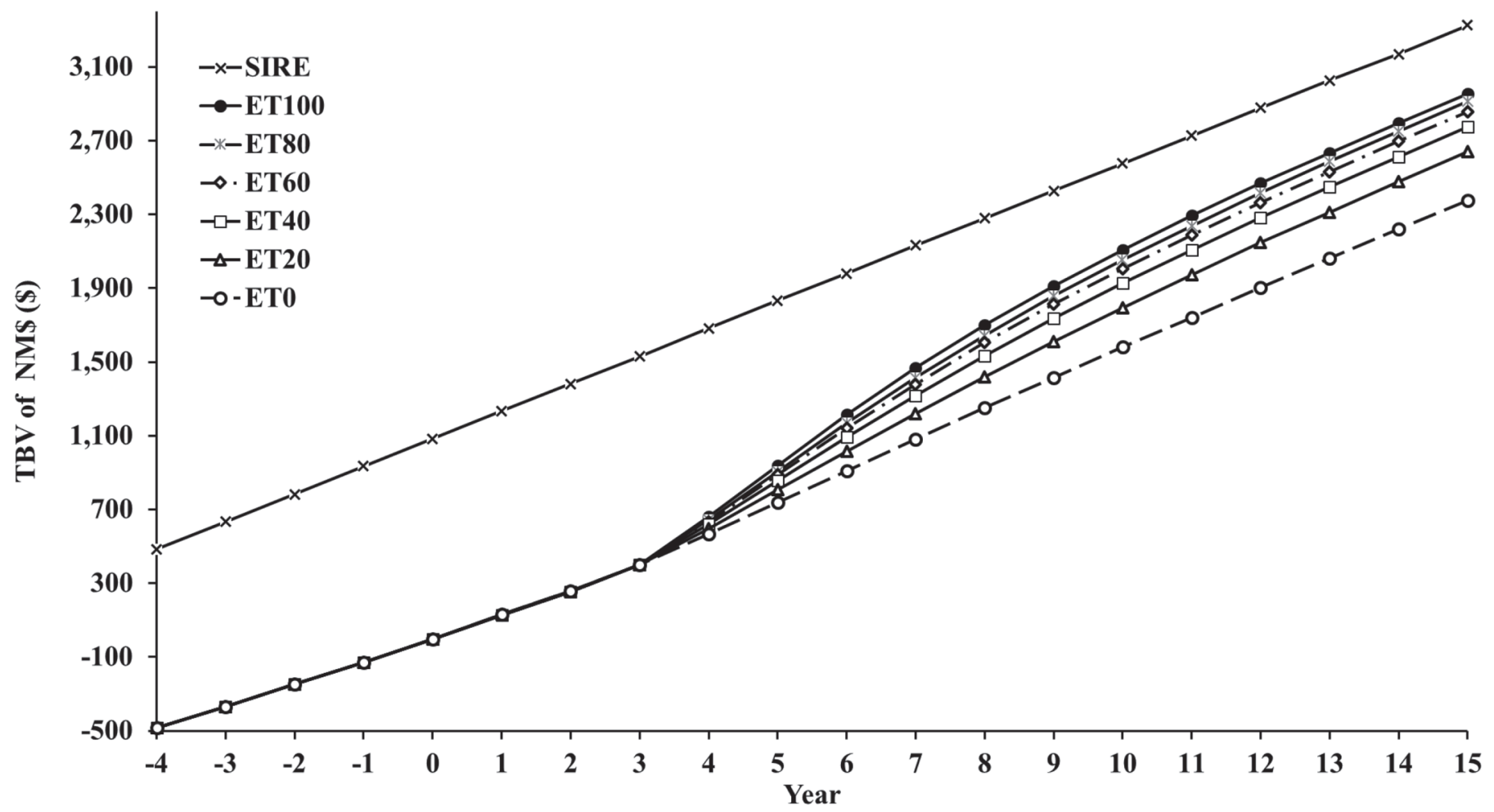

Figure 1. Average true breeding values (TBV) for lifetime net merit $(\mathrm{NM} \$)$ in sires and cows in yr -4 to +15 , in scenarios that used in vitro-produced embryo transfer (IVP-ET) to obtain varying proportions of conceptions from IVP-ET, ranging from no use (ET0) to exclusive use (ET100) of IVP-ET.

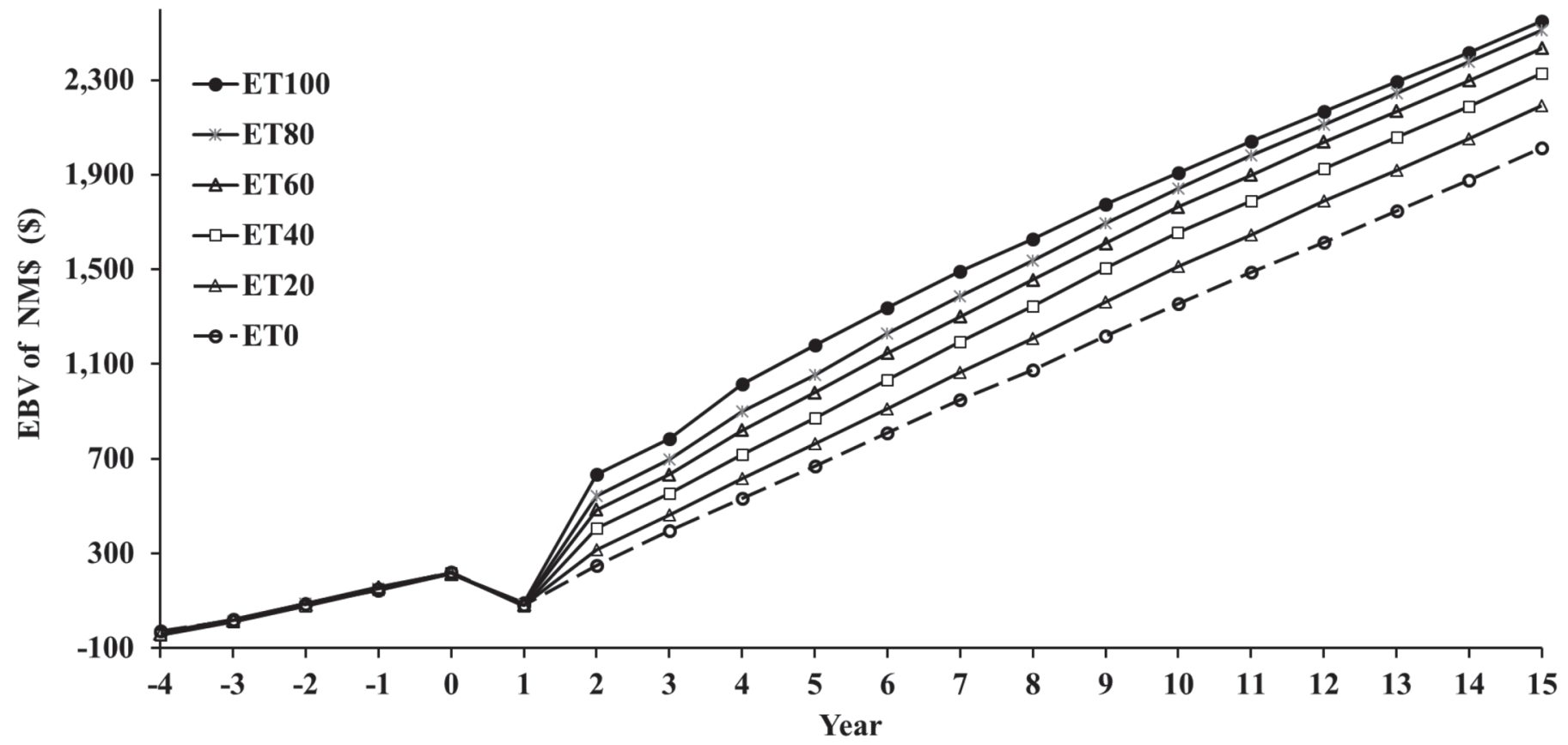

Figure 2. Average EBV of lifetime net merit $(\mathrm{NM} \$)$ for sold surplus dairy heifer calves in yr -4 to +15 , in scenarios that used in vitroproduced embryo transfer (IVP-ET) to obtain varying proportions of conceptions from IVP-ET, ranging from no use (ET0) to exclusive use (ET100) of IVP-ET. The decrease in EBV of $\mathrm{NM} \$$ in yr 1 is due to the increase in reliability to calculated EBV through the introduction of genomic testing at the start of yr 1 . 
responded to the highest TBV of NM $\$$ in ET100. Dairy bull calf sales $(\$ 27)$ were also lowest because approximately $90 \%$ of the calves born were females due to the exclusive use of sexed semen in the IVP-ET scenarios. The financial performance was also calculated when dairy heifer calves were sold at a premium price, which was based on their EBV of NM\$ (Figure 2). Dairy bull and dairy heifer calf sales at premium prices were $\$ 440$ / cow per year for ET100 in yr 15. Milk sales, calf sales, and profit deviation all decreased with less use of IVPET. Cow sales were the lowest in the ET30 scenario. Revenues increased at an average rate of $\$ 67 /$ cow per year per 10 percentage point increase in the proportion of IVF-ET pregnancies from ET0 to ET40 and at an average rate of $\$ 39$ thereafter (Table 3).

Costs. As the proportion of IVP-ET increased, the breeding cost increased and hence the ET100 scenario had the highest breeding cost of $\$ 405 /$ cow per year in yr 15 (Table 3). The difference in breeding cost between ET100 and ET0 was $\$ 358(\$ 405-\$ 47)$ per cow per year. Thus each, 10 percentage point increase in the proportion of IVF-ET pregnancies increased breeding cost by $\$ 36 /$ cow per year. Heifer raising cost was also in general higher with increasing proportions of IVP-ET, as $90 \%$ of the calves born from IVP-ET were females. All dairy heifer calves were raised until the genotyping results were available at approximately $105 \mathrm{~d}$ of age. Hence the variable cost in the ET100 scenario was $\$ 535 /$ cow per year greater than in the ET0 scenario in yr 15 (Table 3), with an average increase of $\$ 54$ in variable cost per 10 percentage points increase in IVFET conceptions. Feed cost increased with greater use of IVP-ET because of greater milk yield.

Profit. Profit per cow per year was $\$ 477$ in yr 0 (Table 3) and increased by at least $\$ 969$ by yr 15 because of increased cow performance. Input prices were kept the same. The ET10 scenario was the most profitable when surplus dairy heifer calves were sold at base prices using the default prices for embryos and surplus dairy heifer calves. The ET40 scenario was the most profitable when surplus dairy heifer calves were sold at premium prices..

Figure 3 shows profits per cow per year over a $15-\mathrm{yr}$ period with surplus dairy heifer calves sold at premium prices for the scenarios ET0, ET40, ET80, and ET100. The figure shows decreases in profit in the initial years for all scenarios where IVP-ET was used due to higher breeding cost per embryo compared with the cost of AI. Profit differences that results from genetic differences in embryos are expected approximately $3 \mathrm{yr}$ after the breeding. Figure 3 shows that the ET100 scenario had the greatest initial decline in profit due to the highest breeding cost. By yr 9 the profit from scenarios with some use of IVP-ET (except for ET100) was greater than the profit from ET0. Results were back in steady state by approximately yr 9 after the introduction of the IVP-ET programs. The profits in yr 15 shown in Figure 3 equal those in Table 3 for the premium dairy heifer calf prices.

Year 15 break-even embryo prices to set profit equal to that obtained with no use of IVP-ET (ET0) are in Table 3. Break-even prices declined with greater use of IVP-ET. Premium prices for sold dairy heifer calves increased the break-even prices for embryos. For example, the break-even embryo price in the ET10 scenario was $\$ 310$ (premium pricing, Table 3). The scenario with the highest break-even embryo price did not result in the maximum profit, however.

The NPV of yr 15 profit for ET40 was $\$ 811 /$ cow per year at a $5 \%$ discount rate. The NPV of the difference in yr 15 profit between ET40 (optimal profit at yr 15) and ET0 was $\$ 33$ per cow. Profit per cow per year of ET40 and ET50 was not different $(P>0.05)$ in scenarios where female calves were sold at premium price. If calves are not sold at premium price, ET40 and ET50 profits are different.

Figure 4 shows the cumulative discounted profit differences per cow from the IVP-ET proportion, which yielded the maximum profit per cow per year in yr 15 for 5 different fresh embryo prices versus the ET0 scenario. The cumulative discounted profit difference from the ET40 scenario at a $\$ 100$ embryo price was $\$ 136$ per cow greater than that from ET0 in yr 15. Break-even cumulative discounted profit occurred in yr 13. Similarly, the cumulative discounted profit difference from the ET100 scenario at a $\$ 50$ embryo price was $\$ 928$ per cow greater than that from ET0 in yr 15. Break-even occurred in yr 9. Use of IVP-ET was not profitable compared with only AI (ET0) in any scenario if the breeding cost per embryo was greater than $\$ 153$ and the proportion of IVP-ET remained constant during the $15 \mathrm{yr}$ in each scenario.

\section{Sensitivity Analysis}

Sensitivity analyses with variations in surplus dairy heifer calf prices and embryo prices aimed to find the optimal proportion of IVP-ET in each price set in yr 15 (Table 4). Profitability results for ET0, ET20, ET40, ET60, ET80, and ET100 are also given. The actual proportion of pregnancies from IVP-ET differed slightly from the intended proportion (e.g., 42 vs. $40 \%$ ) because of discrete donor usage patterns. Greater use of IVPET was profitable when the embryo price was lower and the price for surplus dairy heifer calves was higher. Adding the premium to the surplus dairy heifer calf 
OPTIMAL PROPORTION OF EMBRYO TRANSFER

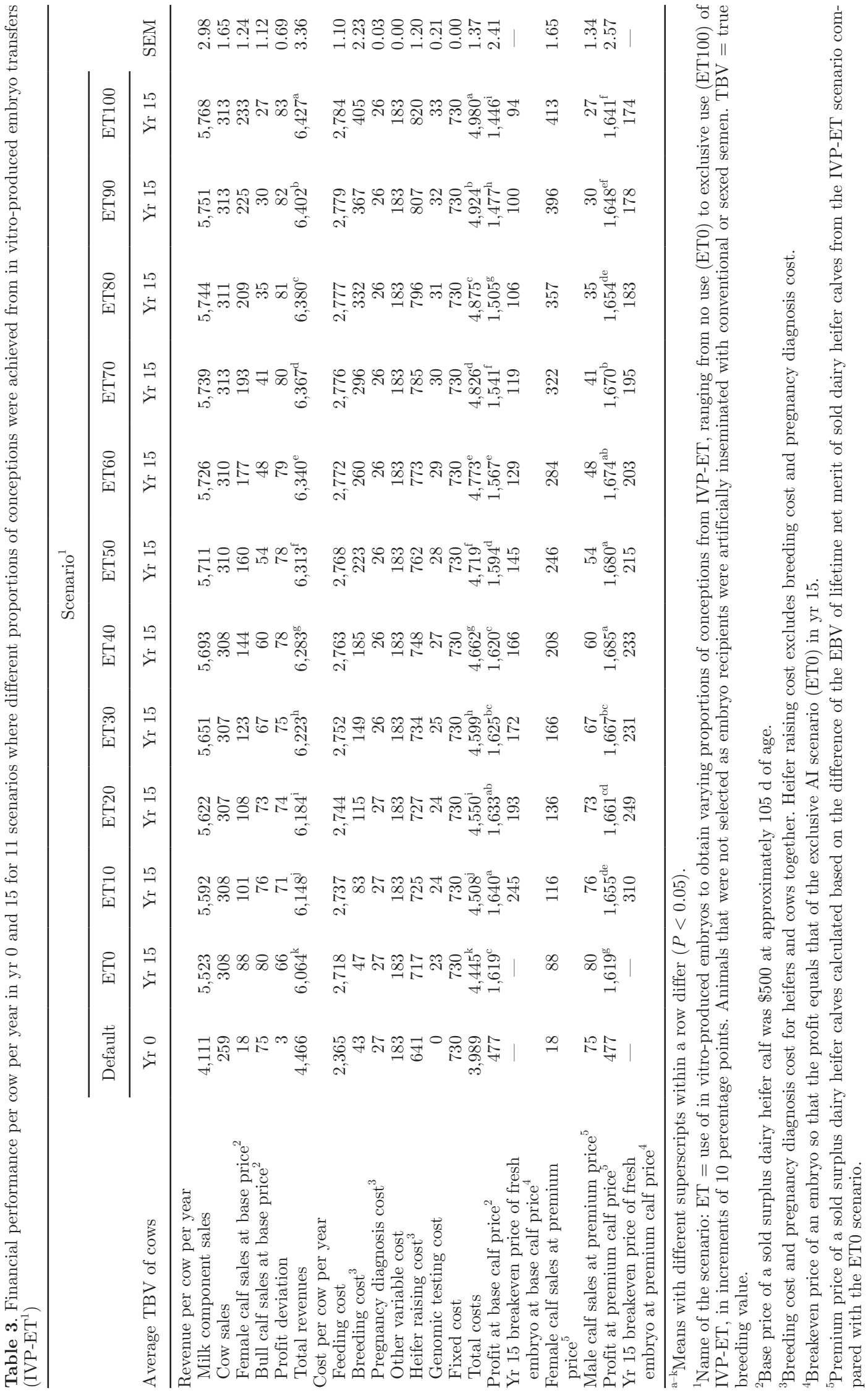




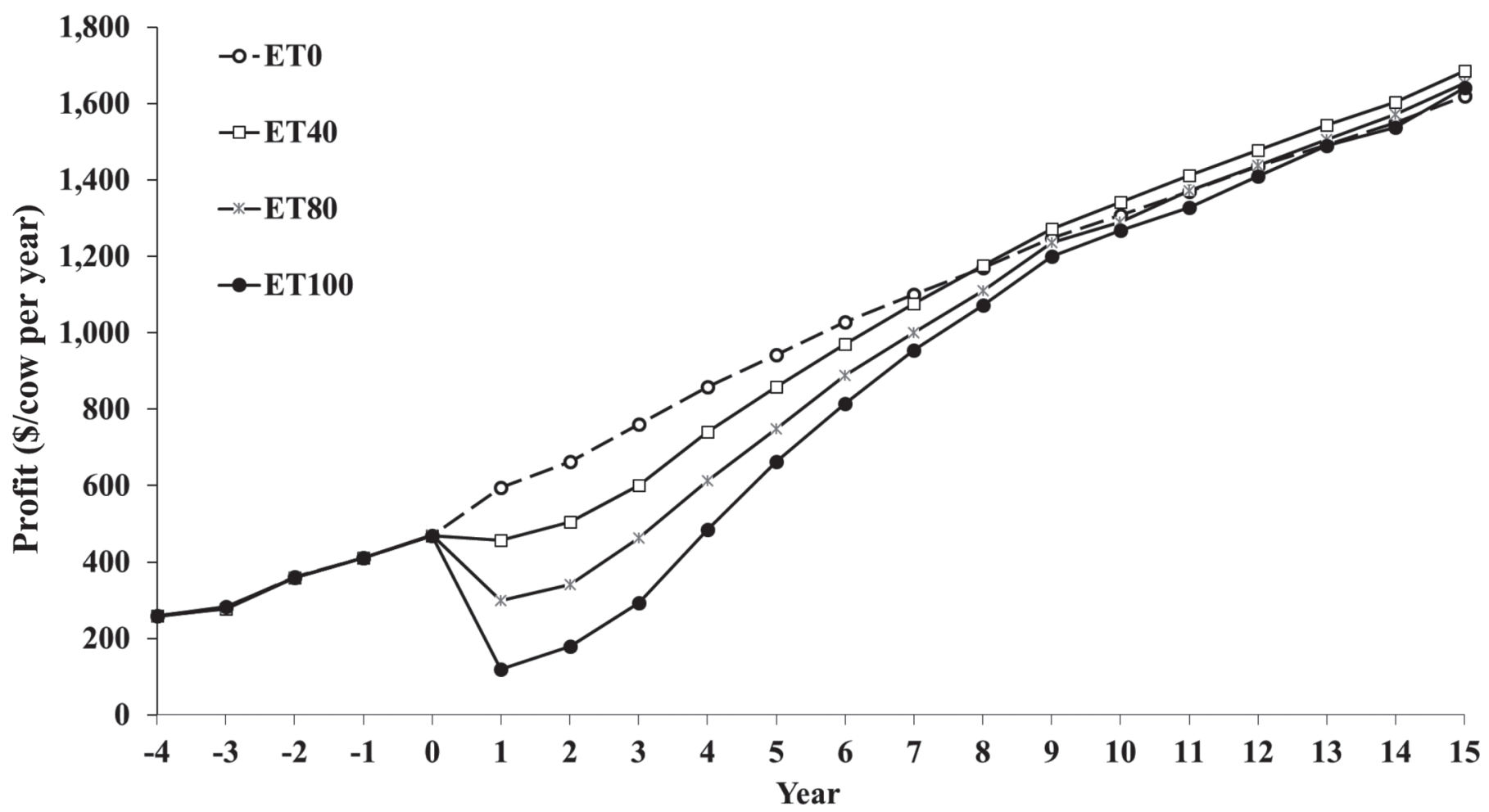

Figure 3. Profit per cow per year in yr -4 to +15 , in scenarios that used in vitro-produced embryo transfer (IVP-ET) to obtain varying proportions of conceptions from IVP-ET, ranging from no use (ET0) to exclusive use (ET100) of IVP-ET. The cost of the fresh embryo was $\$ 165$ and the sale price of a surplus dairy heifer calf was $\$ 500$ in addition to a premium price calculated based on the difference of the estimated breeding value of lifetime net merit of sold dairy heifer calves from the IVP-ET scenario compared with the ET0 scenario.

price added $\$ 28, \$ 65, \$ 108, \$ 149$, and $\$ 195$ to the profit per cow per year for ET20, ET40, ET60, ET80, and ET100, respectively. At a base price of $\$ 300$ for surplus dairy heifer calves and an embryo price of $\$ 50$, the optimal proportion of IVP-ET pregnancies was $46 \%$. Profit per cow per year was $\$ 91$ greater than when only AI was used (ET0). The optimal proportion of IVFET pregnancies increased to $100 \%$ when the embryo price was $\$ 50$ and premium dairy heifer calf pricing was applied, regardless of the base price. Producing at least $10 \%$ of pregnancies from IVP-ET was financially justified in 22 of the 24 scenarios.

\section{DISCUSSION}

The availability of low-cost genomic testing and sexed semen for dairy farms has paved new ways to increase the contribution of the female selection pathway and further reduce the genetic lag with service sires (De Vries et al., 2008; Hjortø et al., 2015). An example is the transfer of IVP embryos from dams identified with genomic testing (Weigel et al., 2012). Some use of IVP-ET was financially warranted for most of the price sets we evaluated. As expected, lower IVP-ET cost and higher prices for surplus dairy heifer calves favored more IVP-ET use.

\section{Genetic Performance of Different IVP-ET Scenarios}

The ET100 scenario maximized the selection intensity and minimized the generation interval among the 11 scenarios. The rate of genetic change in scenario ET100 from yr 1 to 2 was the greatest for the 13 traits toward the selection goal of NM\$. After approximately yr 2, the rates of genetic change were similar among the various scenarios. This implies that the smallest genetic lag with the service sire was obtained with ET100. Over time, the genetic lag in the herd is constant compared with the genetic merit of the service sires when no changes in the reproductive program are made, such as a change from all AI to partial IVP-ET. The level and genetic trend of the service sires (VanRaden and Cole, 2014) was an input in our model and did not depend on the IVP-ET scenario in the dairy herd. No selective mating of sires that match best with the genetic characteristics of the dam was modeled.

The genetic lag between cows and service sires is proportional to the selection intensity, which is affected 


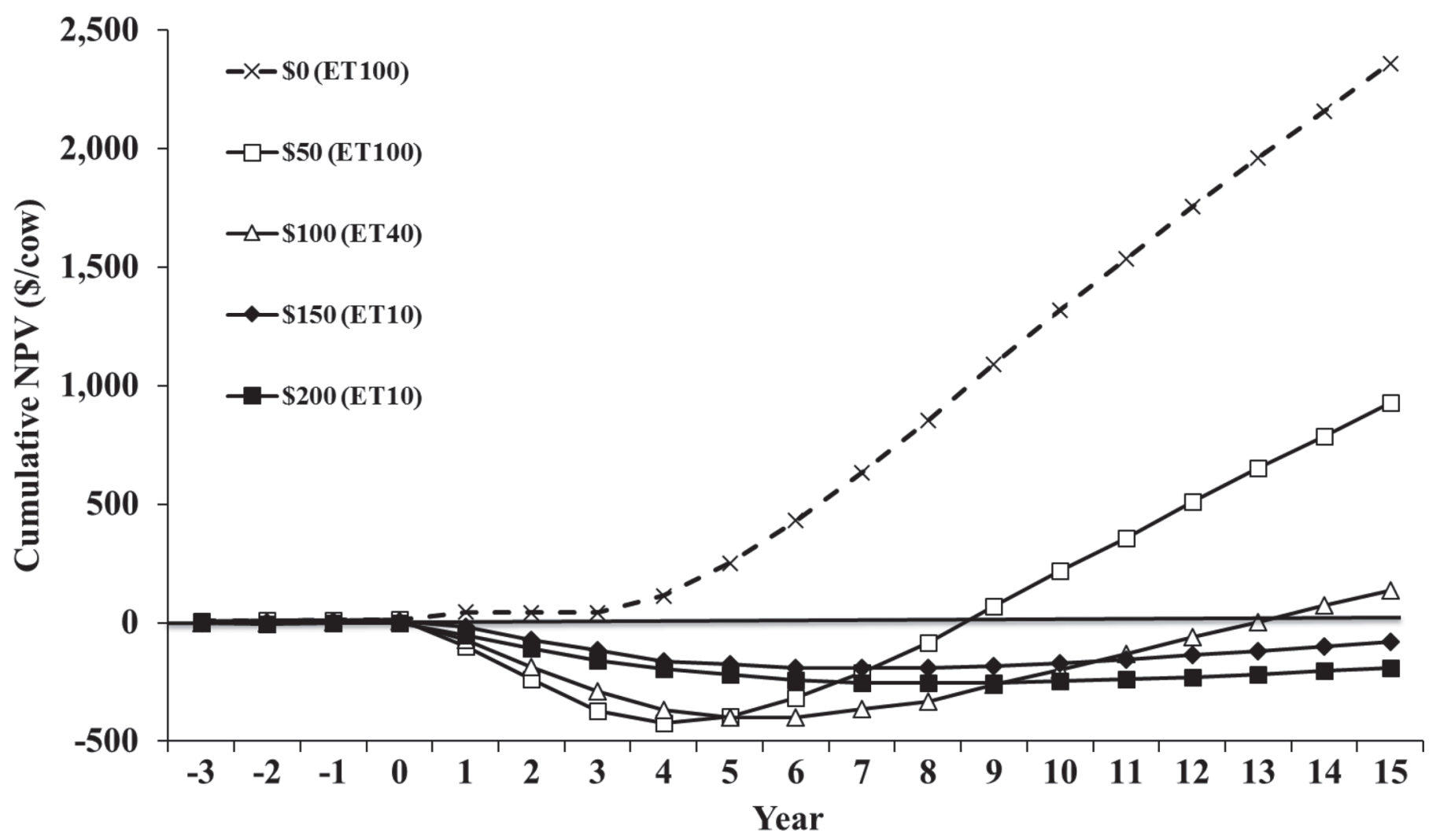

Figure 4. Net present values (NPV) of the cumulative profit difference per cow between the exclusive conventional semen scenario (ET0) and the optimal in vitro-produced embryo transfer (IVP-ET) scenario for a given embryo price. Premium pricing of surplus dairy heifer calves is assumed. Scenarios combined AI and IVP-ET to obtain varying proportions of conceptions from IVP-ET, ranging from no use (ET0) to exclusive use (ET100) of IVP-ET.

by 2 selection factors. First, selection occurs by obtaining a greater proportion of dairy heifer calves from the best dams ranked for EBV of NM\$. In the ET100 scenario, all dairy heifer calves were obtained from 20 of the approximately 180 breeding eligible heifers and cows $(11 \%)$ on the day of donor selection. The second selection occurs by culling the lowest ranked surplus dairy heifer calves on $\mathrm{EBV}$ of $\mathrm{NM} \$$, after retaining the required number of replacement calves. In the ET100 scenario, $46 \%$ of dairy heifer calves were kept to be raised as replacement heifers. Combined, the proportion of dairy heifer calves selected to produce offspring was $46 \% \times 11 \%=5.1 \%$.

The genetic lag further depends on the accuracy of selection. We used a genomic accuracy after genomic testing of all dairy heifer calves. More optimal genomic testing schemes are conceivable, such as a cheaper low accuracy test to identify candidate donors, followed by a higher accuracy test on the candidate donors (Weigel et al., 2012). Separate genomic testing to identify the recipients may also be feasible. We did not explore the financial performance of such alternative schemes.
Finally, the genetic lag with the service sires also depends on the age of the biological dam of the dairy calf. The age of the average dam at time of conception in the ET100 scenario was $597 \mathrm{~d}$ (1.6 yr) younger than in the ET0 scenario.

\section{Phenotypic Performance of Animals Born from Embryo Transfer}

The phenotypic performance of the animals in the model did not depend on the method by which the calves were derived (IVP-ET vs. AI) or the type of semen sorting (sorting before freezing, or reverse sorted). Siqueira et al. (2017) reported that dairy heifer calves derived by IVP were born slightly heavier compared with AI calves. Cumulative mortality of calves derived by IVP with reverse female-sorted semen was greater by $180 \mathrm{~d}$ of age compared with AI offspring. Mortality of calves derived by IVP with conventional semen was not different from those derived by AI, however. In their study, cows derived by IVP with reverse femalesorted semen produced less milk, fat, and protein in 
KANIYAMATTAM ET AL.

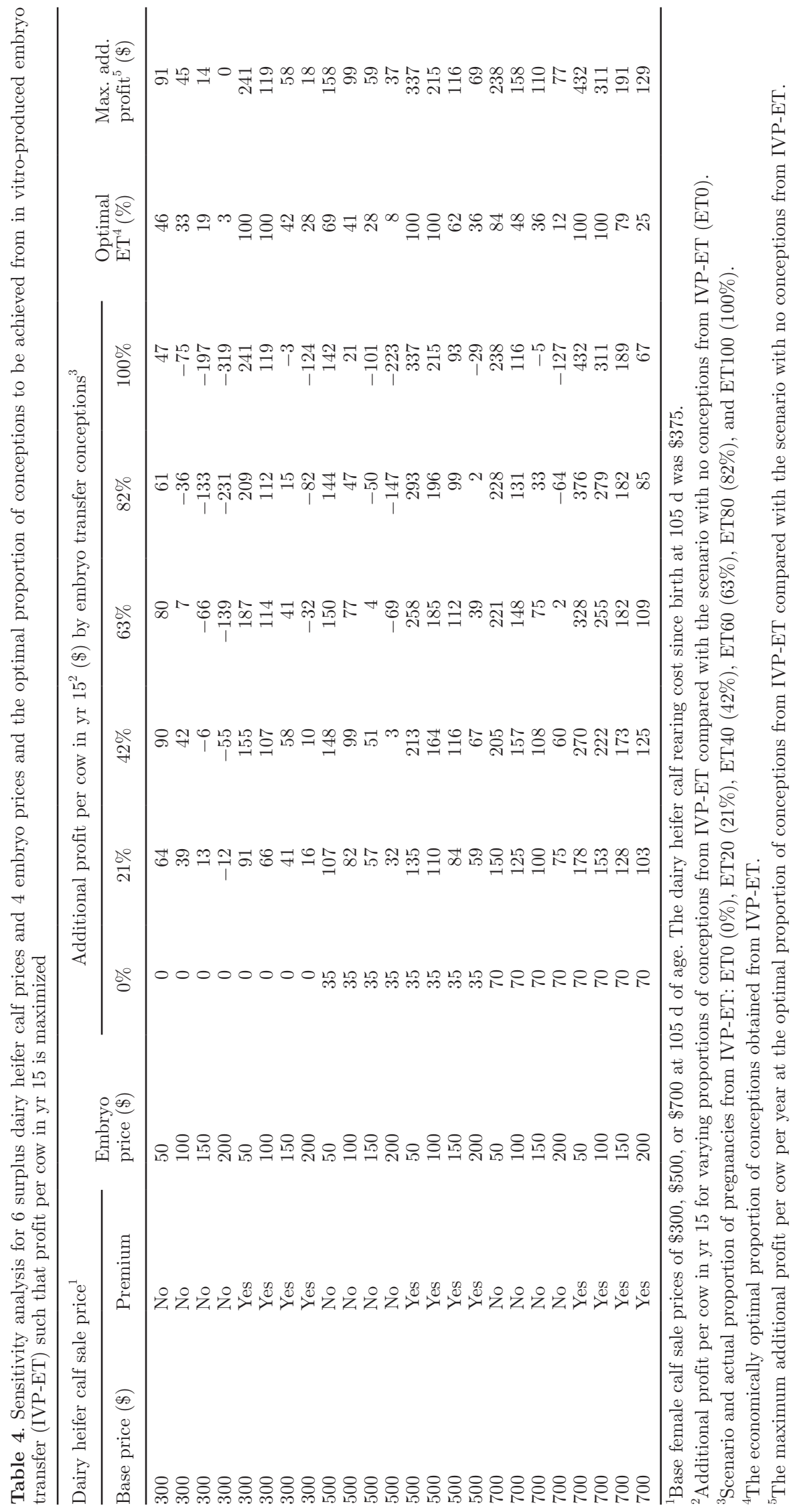


their first lactation compared with dairy cows derived by AI. Collectively, these effects would reduce the value of dairy heifer calves from IVP-ET compared with AI.

\section{Selection of Recipients}

In the current simulation, recipients were selected based on fertility traits present in the $\mathrm{NM} \$$ index. Motivation was the greatest predicted probability of pregnancy per breeding given the high price of transferred embryos. Better criteria to select recipients may exist. An alternative simulation was also conducted in which cow recipients were ranked based on milk yield to avoid culling due to low milk yield (data not shown). Eligible heifer recipients were ranked as described earlier. In this strategy, the average TBV of milk yield of cows in the herd was $45 \mathrm{~kg} / 305$ d lower for the ET30 to ET80 scenarios as compared with rankings based on fertility traits (first strategy). The average TBV of DPR, CCR, and heifer conception rate was on average $0.3,0.6$, and 0.15 percentage points higher in scenarios ET30 to ET80 (data not shown) than in the same scenarios in which cow recipients were selected based on fertility traits (first strategy). Hence, the recipient selection strategy affected the genetic merit of the herd in a significant manner.

Phenotypic indications of fertility are likely important too when identifying recipients. An example may be quality of display of estrus, possibly supported with decision aids such as accelerometers (e.g., Silper et al., 2015; Pereira et al., 2016). Another example is that cows that experienced dystocia and lost much body condition are known to have lower fertility (Berry et al., 2007). Other unknown factors play a role in the success of the recipient to produce a live calf after ET such that recipients that have successfully carried an ET calf before could be used again (Peterson and Lee, 2003).

Genetic merit for stillbirth, included in the EBV for calving ability, could also be sufficiently important to consider when selecting recipients. In addition, future performance of the embryo after birth, such as reduced milk yield depending on recipient parity, should be considered (Siqueira et al., 2017). Collectively, the optimal selection of eligible recipients considering all relevant factors remains an open question.

\section{Influence of Input Assumptions on Financial Performance of IVP-ET Scenarios}

The financially optimal proportion of IVP-ET conceptions calculated for 6 surplus dairy heifer calf prices and 4 embryo prices showed that some use of IVP-ET was profitable at embryo prices that ranged from $\$ 50$ to $\$ 200$ and for realistic dairy heifer calf prices.
Economic studies in the 1980s concluded that ET was too expensive at that time for commercial dairy farmers to replace culled cows (Ferris and Troyer, 1987). Based on genetic change in milk yield only, Ferris and Troyer (1987) calculated break-even prices per ET of $\$ 24$ to $\$ 125$ (\$52 to $\$ 269$ in 2017 ). These authors also noted that the early years after the start of the ET program had expenses but no returns to better genetics yet, and therefore that the planning horizon of the use of ET was important when calculating economic feasibility. Later, Smeaton et al. (2003) concluded that the profitability of embryo-based reproductive technologies could occur only if prices per pregnancy are less than 2 to 4 times greater than prices of AI or natural matings. Ghavi Hossein-Zadeh et al. (2011) also found that a significant decrease in cost per ET and an increase in the ET performance are necessary for ET to become profitable. These authors found that the use of ET was more valuable in expanding herds than in constant herd sizes. Assumptions in all these studies were different from our study, such as selection criteria, use of genomic testing, sale of surplus dairy heifer calves, and conception rates of transferred embryos. Our results suggest that sufficient progress has been made to reduce costs such that IVP-ET could now be financially warranted on many dairy farms.

The results for the various fractions IVP-ET in yr 10 to 15 were approximately constant. The optimal fraction IVP-ET is therefore likely fairly similar over time once steady state is reached again. This implies that the optimal fraction IVP-ET is more dependent on the genetic lag between service sire and dam than on the absolute level of genetic merit of the service sire and dam.

The scenario with a $\$ 500$ base price for a sold dairy heifer calf without premium (\$125 profit over rearing costs) and a $\$ 150$ embryo price is a realistic scenario today (yr 0). In this case, the optimal fraction IVP-ET was $28 \%$ with an additional profit of $\$ 59$ per cow per year compared with use of AI only.

Adding a premium based on the genetic merit to the value of the sold surplus dairy heifer calf increased the optimal fraction of IVP-ET. Such premiums are not common today, but it is plausible that dairy heifer calf buyers are willing to pay premiums when genetic merit has consequences for the value of the surplus dairy heifer calf in the future. An example is when such calves are raised to become dairy cows on another dairy farm.

In the current study, the proportion of IVP-ET remained the same over time, which let to low profitability in the early years after the start of the IVP-ET program, especially when the fraction of IVP-ET use was high. An improved scheme could be a gradual increase in the fraction of IVP-ET use over time. The 
optimal speed of introduction of IVP-ET in the herd depends on the herd's goals, such as limiting a period of low cash flow or fast genetic progress. It is also possible that the optimal fraction of IVP-ET is reduced again once the genetic lag with the service sires has been decreased. The best introduction scheme of IVP-ET in a herd is a topic for further research.

Embryo transfer cost and value of surplus dairy heifer calves are major determinants of the value of IVP-ET, but other factors may play an important role as well. A global sensitivity analysis (e.g., Saltelli et al., 2008) to determine these major factors was not carried out.

None of the input prices for milk, feed, calves, replacement heifers, and so on used to calculate the profitability of the various scenarios were identical to those used in the NM\$ index that we used to select donors and sell surplus dairy heifer calves. Exceptions were the 4 profit deviations we used to value 4 of the 12 traits. Therefore, financially better indices exist. Use of such an index might value embryos from IVP versus AI differently than in the current study.

Input prices also have a direct effect on the optimal proportion of IVP-ET. For example, in yr 0 the milk sales minus feed cost per cow per day (income over feed cost; IOFC) was $\$ 5.80$, which is below the 25 th quartile of IOFC for US dairy farms for 2004 to 2015 (Gould, 2016). Therefore, the IOFC in yr 15, which was in a range of $\$ 9.20$ to $\$ 10.30$ for ET0 to ET100, was based on a conservative estimate of $\$ 5.80$ from yr 0 . The difference of $\$ 10.30-\$ 5.80=\$ 4.50$ for ET100 is entirely the economic benefit of genetic gain achieved by the exclusive use of IVP-ET. In case the future average IOFC is higher than $\$ 5.80$, the profit from greater use of IVP-ET will likely be higher.

Seasonality of performance of dairy cattle was also not included in our study. Reduced conception rates because of summer heat stress would likely increase the use of IVP-ET in the summer because pregnancy rates from IVP-ET are less affected by heat stress than pregnancy rates by AI (Stewart et al., 2011; Kadokawa et al., 2012). The optimal use of IVP-ET in seasonal herds remains an open question.

The annual cow cull rate was kept constant in our study and is unlikely to be financially optimal. We did not attempt to find an optimal culling policy and cull rate for the various combinations of IVP-ET scenarios and prices. Increased rate of genetic change from dairy heifers compared with older cows in the herd should increase cow cull rates, but the influence of genetic progress on optimal cull rates appears to be minor (De Vries, 2017).

The use of sexed semen was limited to heifers and was not varied in our study. More or less use of sexed semen will affect the optimal use of IVP-ET. We also did not investigate the effect of using beef semen to derive crossbred calves when calculating the optimal proportion of IVP-ET. Crossbred dairy-beef calves may be sold for higher prices than purebred dairy calves. Ettema et al. (2017) studied the economic opportunities of combining sexed semen with beef semen (all AI) to derive just enough dairy heifer calves to replace culled cows. They reported that the combined use of beef and sexed semen could decrease genetic lag, but none of the scenarios were profitable under Danish circumstances when the value of the decreased genetic lag was not included. Depending on markets for pure and crossbred calves, best strategies given the availability of IVP-ET, and sexed, conventional, and beef semen should be investigated. Changes in genetic merit should be included. Finally, the multi-trait architecture of the model in this study allows for easy inclusion of additional traits such as livability (VanRaden, 2017), feed efficiency (VandeHaar et al., 2016), or traits related to numbers of oocytes collection, cleaved embryos, embryo quality, and transferrable embryos (Parker Gaddis et al., 2017).

\section{CONCLUSIONS}

We adjusted a previously developed stochastic, dynamic dairy model with multi-trait genetics that includes the genetic, technical, and financial performance of dairy herds that use different proportions of pregnancies generated by IVP-ET and AI. The yr 15 profit after the start of the IVP-ET program was maximized when $40 \%$ of the total pregnancies in the herd came from IVP-ET. Lower prices for IVP-ET or greater values of surplus dairy heifer calves increased the optimal use of IVP-ET. Some use of IVP-ET was profitable in many realistic combinations of embryo prices and surplus dairy heifer calf values.

\section{ACKNOWLEDGMENTS}

This study was financially supported by USDA National Institute Food and Agriculture, Agriculture and Food Research Initiative grant award 2013-68004-20365 titled "Improving Fertility of Dairy Cattle Using Translational Genomics."

\section{REFERENCES}

Beltrame, R. T., L. G. Barioni, B. D. Maestri, and C. R. Quirino. 2007. Economic optimization of the number of recipients in bovine embryo transfer programs. Sci. Agric. 64:221-226.

Berry, D. P., J. M. Lee, K. A. Macdonald, and J. R. Roche. 2007. Body condition score and body weight effects on dystocia and stillbirths and consequent effects on postcalving performance. J. Dairy Sci. 90:4201-4211. 
Block, J., L. Bonilla, and P. J. Hansen. 2010. Efficacy of in vitro embryo transfer in lactating dairy cows using fresh or vitrified embryos produced in a novel embryo culture medium. J. Dairy Sci. 93:5234-5242.

Bonilla, L., J. Block, A. C. Denicol, and P. J. Hansen. 2014. Consequences of transfer of an in vitro-produced embryo for the dam and resultant calf. J. Dairy Sci. 97:229-239.

Chaubal, S., J. Molina, C. Ohlrichs, L. Ferre, D. Faber, P. Bols, J. Riesen, X. Tian, and X. Yang. 2006. Comparison of different transvaginal ovum pick-up protocols to optimize oocyte retrieval and embryo production over a 10 -week period in cows. Theriogenology 65:1631-1648.

De Vries, A. 2017. Economic trade-offs between genetic improvement and longevity in dairy cattle. J. Dairy Sci. 100:4184-4192.

De Vries, A., M. Overton, J. Fetrow, K. Leslie, S. Eicker, and G. Rogers. 2008. Exploring the impact of sexed semen on the structure of the dairy industry. J. Dairy Sci. 91:847-856.

Ettema, J. F., S. Østergaard, and M. K. Sørensen. 2011. Effect of including genetic progress in milk yield on evaluating the use of sexed semen and other reproduction strategies in a dairy herd. Animal 5:1887-1897.

Ettema, J. F., J. R. Thomasen, L. Hjortø, M. Kargo, S. Østergaard, and A. C. Sørensen. 2017. Economic opportunities for using sexed semen and semen of beef bulls in dairy herds. J. Dairy Sci. 100:4161-4171.

Ferris, T. A., and B. W. Troyer. 1987. Break-even costs for embryo transfer in a commercial dairy herd. J. Dairy Sci. 70:2394-2401.

Ghavi Hossein-Zadeh, N., A. Nejati-Javaremi, S. R. Miraei-Ashtiani, H. Kohram, and M. Honarvar. 2011. Bio-economic model to evaluate twinning rate using sexed embryo transfer in dairy herds. Animal 5:1705-1719.

Gould, B. W. 2016. Understanding Dairy Markets. Dept. of Agric. and Appl. Econ., Univ. Wisconsin. Accessed Jul. 1, 2017. http:// future.aae.wisc.edu/data/monthly_values/by_area/2403?area= US\&grid $=$ true $\&$ tab $=$ costs

Hansen, P. J. 2014. Current and future assisted reproductive technologies for mammalian farm animals. Pages 1-22 in Current and Future Reproductive Technologies and World Food Production. Vol. 752. G. C. Lamb and N. DiLorenzo, ed. 1st ed. Springer, New York, NY.

Hansen, P. J., and J. Block. 2004. Towards an embryocentric world: The current and potential uses of embryo technologies in dairy production. Reprod. Fertil. Dev. 16:1-14.

Heikkilä, A., and J. Peippo. 2012. Optimal utilization of modern reproductive technologies to maximize the gross margin of milk production. Anim. Reprod. Sci. 132:129-138.

Hjortø, L., J. F. Ettema, M. Kargo, and A. C. Sørensen. 2015. Genomic testing interacts with reproductive surplus in reducing genetic lag and increasing economic net return. J. Dairy Sci. 98:646-658.

Kadokawa, H., M. Sakatani, and P. J. Hansen. 2012. Perspectives on improvement of reproduction in cattle during heat stress in a future Japan. Anim. Sci. J. 83:439-445.

Kaniyamattam, K., J. Block, P. J. Hansen, and A. De Vries. 2017. Comparison between an exclusive in vitro produced embryo transfer system and artificial insemination for genetic, technical, and financial herd performance. J. Dairy Sci. 100:5729-5745.

Kaniyamattam, K., M. A. Elzo, J. B. Cole, and A. De Vries. 2016 Stochastic dynamic simulation modelling including multitrait genetics to estimate genetic, technical and financial consequences of dairy farm reproduction and selection strategies. J. Dairy Sci. 99:8187-8202.

Kruip, Th. A. M., R. Boni, Y. A. Wurth, M. W. M. Roelofsen, and M. C. Pieterse. 1994. Potential use of ovum pick-up for embryo production and breeding in cattle. Theriogenology 42:675-684.
Meuwissen, T. 1991. Expectation and variance of genetic gain in open and closed nucleus and progeny testing schemes. Anim. Prod. 53:133-141.

Parker Gaddis, K. L., S. Dikmen, D. J. Null, J. B. Cole, and P. J. Hansen. 2017. Evaluation of genetic components in traits related to superovulation, in vitro fertilization, and embryo transfer in Holstein cattle. J. Dairy Sci. 100:2877-2891.

Pereira, M. H. C., M. C. Wiltbank, and J. L. M. Vasconcelos. 2016. Expression of estrus improves fertility and decreases pregnancy losses in lactating dairy cows that receive artificial insemination or embryo transfer. J. Dairy Sci. 99:2237-2247.

Peterson, A. J., and R. S.-F. Lee. 2003. Improving successful pregnancies after embryo transfer. Theriogenology 59:687-697.

Pryce, J. E., M. E. Goddard, H. W. Raadsma, and B. J. Hayes. 2010. Deterministic models of breeding scheme designs that incorporate genomic selection. J. Dairy Sci. 93:5455-5466.

Ribeiro, E. S., K. N. Galvão, W. W. Thatcher, and J. E. P. Santos. 2012. Economic aspects of applying reproductive technologies to dairy herds. Anim. Reprod. 9:370-387.

Saltelli, A., M. Ratto, T. Andres, F. Campolongo, J. Cariboni, D. Gatelli, M. Saisana, and S. Tarantola. 2008. Global Sensitivity Analysis: The Primer. John Wiley and Sons, Chichester, West Sussex, United Kingdom.

Silper, B. F., A. M. L. Madureira, M. Kaur, T. A. Burnett, and R. L. A. Cerri. 2015. Short communication: Comparison of estrus characteristics in Holstein heifers by 2 activity monitoring systems. J. Dairy Sci. 98:3158-3165.

Siqueira, L. G. B., S. Dikmen, M. S. Ortega, and P. J. Hansen. 2017. Postnatal phenotype of dairy cows is altered by in vitro embryo production using reverse X-sorted semen. J. Dairy Sci. 100:58995908.

Smeaton, D. C., B. L. Harris, Z. Z. Xu, and W. H. Vivanco. 2003. Factors affecting commercial application of embryo technologies in New Zealand: a modelling approach. Theriogenology 59:617-634.

Stewart, B. M., J. Block, P. Morelli, A. E. Navarette, M. Amstalden, L. Bonilla, P. J. Hansen, and T. R. Bilby. 2011. Efficacy of embryo transfer in lactating dairy cows during summer using fresh or vitrified embryos produced in vitro with sex-sorted semen. J. Dairy Sci. 94:3437-3445.

Thomasen, J., A. Willam, C. Egger-Danner, and A. C. Sørensen. 2016. Reproductive technologies combine well with genomic selection in dairy breeding programs. J. Dairy Sci. 99:1331-1340.

VandeHaar, M. J., L. E. Armentano, K. Weigel, D. M. Spurlock, R. J. Tempelman, and R. Veerkamp. 2016. Harnessing the genetics of the modern dairy cow to continue improvements in feed efficiency. J. Dairy Sci. 99:4941-4954.

VanRaden, P. M. 2017. Net merit as a measure of lifetime profit: 2017 revision. AIP Research Report NM\$6 (2-17). Accessed Jun. 16 2017. https://www.aipl.arsusda.gov/reference/nmcalc-2017.htm.

VanRaden, P. M., and J. B. Cole. 2014. Net merit as a measure of lifetime profit: 2014 revision. AIP Research Report NM\$5 (10-14). Accessed May 31, 2017. http://aipl.arsusda.gov/reference/nmcalc $-2014 . \mathrm{htm}$

Weigel, K. A., P. C. Hoffman, W. Herring, and T. J. Lawlor Jr. 2012. Potential gains in lifetime net merit from genomic testing of cows, heifers, and calves on commercial dairy farms. J. Dairy Sci. 95:2215-2225.

Wilson, R. D., P. M. Fricke, M. L. Leibfried-Rutledge, J. J. Rutledge, C. M. Penfield, and K. A. Weigel. 2006. In vitro production of bovine embryos using sex-sorted sperm. Theriogenology 65:10071015 . 